\title{
Multi-stage redox systems based on Dicationic P-containing Polycyclic Aromatic Hydrocarbons
}

\author{
Thomas Delouche, ${ }^{[a]}$ Antoine Vacher, ${ }^{[a]}$ Elsa Caytan,,${ }^{[a]}$ Thierry Roisnel, ${ }^{[a]}$ Boris Le Guennic, ${ }^{[a]}$ Denis \\ Jacquemin, ${ }^{[b] *}$ Muriel Hissler, ${ }^{[a]}$ and Pierre-Antoine Bouit ${ }^{[a] *}$
}
[a] T. Delouche, Dr A. Vacher, Dr E. Caytan, Dr T. Roisnel, Dr B. Le Guennic, Prof. M. Hissler, Dr P. A. Bouit Univ Rennes, CNRS, ISCR - UMR 6226
F-35000 Rennes, France
E-mail: pierre-antoine.bouit@univ-rennes1.fr
[b] Prof. D. Jacquemin
CEISAM UMR CNRS 6230
2, rue de la Houssinière, University of Nantes, Nantes, France.
E-mail: denis.jacquemin@univ-nantes.fr

\begin{abstract}
In this communication, we report the straightforward synthesis of unprecedented electron-acceptors based on dicationic $\mathrm{P}$ containing PAHs (Polycyclic Aromatic Hydrocarbons) based on copper mediated radical approach. In these systems, two phosphoniums are connected through various PAHs backbones. The impact of $\pi$-extension on both the optical and redox properties is investigated using a joint experimental (UV-Vis absorption, fluorescence and cyclic voltammetry) and theoretical approach (DFT calculations). Finally, (spectro)-electrochemical studies prove that these compounds possess three redox states and EPR studies confirms the in situ formation of an organic radical delocalized on the PAH backbone.
\end{abstract}

Organic multi-stage redox systems are key component in many technological fields ranging from organic electronics (solar cells, batteries...) to spintronics. ${ }^{[1]}$ Among the strategies used to design such compounds, one approach consists in linking two cationic heteroatoms through a $\pi$-conjugated backbone featuring an even number of $s p^{2} \mathrm{C}$-atoms (Scheme 1). ${ }^{[2,3]}$ This led to the preparation of "Weitz type" derivatives which display three redox states including a stable radical cation intermediate. ${ }^{[2,3]}$ MethylViologens (MV) are probably the most emblematic electron acceptors of this family. ${ }^{[4,5]}$ Although Hünig et al. mentioned in 1978 the applicability of this general strategy to systems in which phosphorus is the unique heteroatom, ${ }^{[3]}$ such derivatives have not been described yet to the best of our knowledge, despite the wide literature on organophosphorus based $\pi$-systems and switches. ${ }^{[6-}$ ${ }^{8]}$ Due to the specificities of $\mathrm{P}$ compared to the others heteroatoms in terms of electronegativity, valence, coordination number, it appeared interesting to us to study the impact of its insertion on the optical/redox properties. ${ }^{[9]}$

To tackle this challenge, we here take advantage of the possibility to convert a cationic $\sigma^{4}, \lambda^{4}-\mathrm{P}^{+}$into a neutral $\sigma^{4}, \lambda^{5}-\mathrm{P}$ (Scheme 1$)$ to afford multi-stage redox systems based on organophosphorus derivatives. ${ }^{[10]}$ Indeed we report the general and straightforward synthesis of an unprecedented family of dicationic P-containing Polycyclic Aromatic Hydrocarbons (PAHs) 2-6[OTf $]_{2}$ where two $\sigma^{4}, \lambda^{4} \mathrm{P}^{+}$are connected through various $\pi$-backbones (Scheme 2). The impact of $\pi$-extension on both optical and redox properties is investigated using a joint experimental/theoretical approach. Finally, (spectro)-electrochemical studies prove that these compounds possess three redox states and EPR studies confirms the in situ formation of radicals demonstrating that these novel acceptors behave as "viologen-like" redox systems. ${ }^{4,6 e]}$

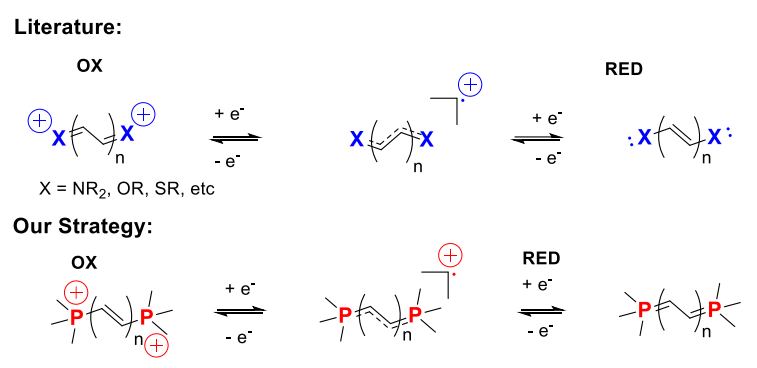

Scheme 1. General structure of multi-stage redox systems ${ }^{[3]}$ and their corresponding phosphorus containing analogs

To prepare the targeted bisphosphoniums, we rely on a coppermediated radical approach. ${ }^{[11]}$ This method was recently used to prepare $\pi$-conjugated phosphoniums. ${ }^{[12]}$ In our hands, the double phosphacyclization of bisphosphine 1 cleanly afforded 1,8bisphosphapyrenium 2[OTf $]_{2}$ (87\% yield, Scheme 2), as an air and moisture stable derivatives characterized by a single peak in ${ }^{31} \mathrm{P}$ NMR $(\delta=+1.3 \mathrm{ppm})$. This compound was fully characterized by multinuclear NMR, high-resolution mass spectrometry and $\mathrm{X}$ ray diffraction (vide infra). In addition, 2[OTf $]_{2}$ is soluble in classical organic solvents. To illustrate the versatility of this molecular engineering strategy, various polyaromatic diphosphines were converted into their dicationic analogs 3$6[\mathrm{OTf}]_{2}$ in good yields (Scheme 2). All the precursors were synthesized through lithiation of the corresponding dibromoderivatives which were then reacted with chlorodiphenylphosphine (see ESI). This allowed us to prepare a novel family of dicationic P-containing PAHs featuring variable $\pi$ cores. They all display two cationic $\sigma^{4}, \lambda^{4}$-P centers connected through a bridge featuring an even number of $s p^{2} \mathrm{C}$-atoms. 


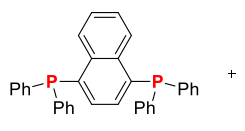

1
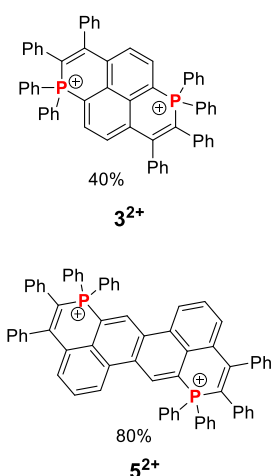

$5^{2+}$

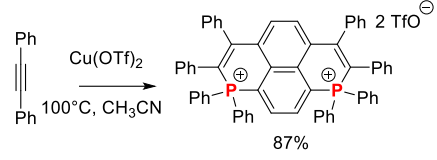

$2[\mathrm{OTf}]_{2}$

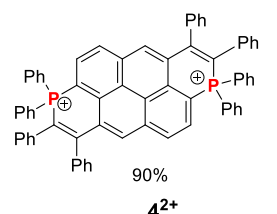

$4^{2+}$

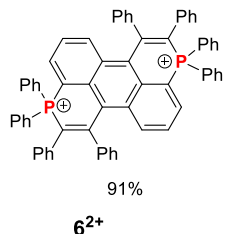

$6^{2+}$
Scheme 2. Synthetic access to 2[OTf $]_{2}$ and chemical structure of compounds $3-6^{2+}$ (TfO- anions have been omitted for clarity)

The structures of derivatives 2-6[OTf $]_{2}$ were all confirmed by single-crystal X-ray diffraction (Fig. 1, Table S1-2). In all compounds, the $\sigma^{4}, \lambda^{4}-\mathrm{P}$ atom shows a tetrahedral shape with usual valence angles and C-P bond lengths. In 2-5[OTf $]_{2}$, the polyaromatic scaffold featuring the $\mathrm{P}$-heterocycles is mainly planar (maximum deviation from the mean C-sp plane, $0.32 \AA$ ), and the lateral phenyl rings lie perpendicularly to the main plane. The case of bisphosphaperylenium $6[\mathrm{OTf}]_{2}$ is more peculiar as a significant $\pi$-curvature is observed (maximum deviation from the mean C-sp $p^{2}$ plane, $0.78 \AA$, Fig. S1) due to steric hindrance between $\mathrm{H}$ and $\mathrm{Ph}$ at the bay position. ${ }^{[13]}$ This structural feature of the solid state pertains in solution according to DFT calculations (Fig. S17-19). Finally, no intermolecular contact could be observed in the packing, which most likely results from the presence of bulky tetrahedral P-atoms, lateral exocyclic phenyl rings, and counterions.

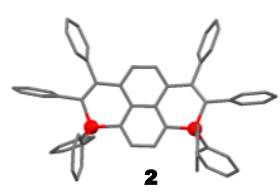

$\mathbf{P}$
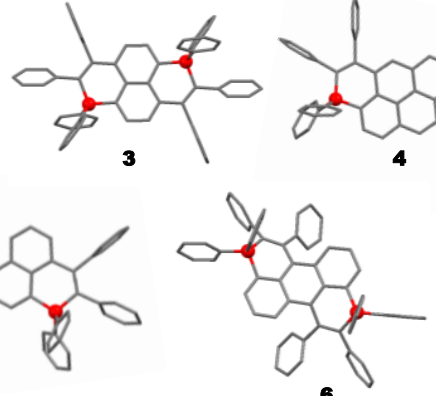

5

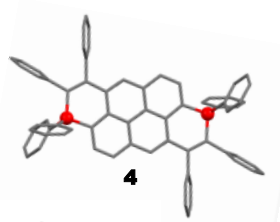

Figure 1. X-ray crystallographic structure of $2-6^{2+}(\mathrm{H}$ atoms, counterions and solvent omitted for clarity).

The spectroscopic properties of 2-6[OTf $]_{2}$ are investigated in diluted $\mathrm{CH}_{2} \mathrm{Cl}_{2}$ solutions ( $\mathrm{c}=5.10^{-6} \mathrm{~mol} . \mathrm{L}^{-1}$, Fig. 2 and Table S3). 2-5[OTf $]_{2}$ display structured absorption bands in the same window with $\lambda_{\text {abs }}$ spanning from 417 to $432 \mathrm{~nm}$. This absorption can be attributed to a delocalized $\pi-\pi^{*}$ transition according to TD-DFT calculations (Fig 2 and Fig. S13). While the size of the polycyclic scaffold and the relative position of the $\mathrm{P}$-atoms weakly affect the maximum absorption wavelength, the vibronic progression and the extinction coefficient are more dependent on the structure of the molecule. In contrast, $6[\mathrm{OTf}]_{2}$ displays a marked bathochromic shift compared to $2[\mathrm{OTf}]_{2}(\Delta \lambda=102 \mathrm{~nm})$. These results illustrate that the maximal wavelength of absorption of such compounds cannot be easily rationalized by comparing the $\pi$-extension. In this case, it is determined by a complex interplay between the $\pi$ extension, the relative P-positions and the $\pi$-curvature. ${ }^{[14]}$ The qualitative evolutions in the 2-6[OTf $]_{2}$ series are reasonably reproduced by TD-DFT (Table S3). Interestingly, the $\mathrm{P}$ centers play a significant role in the transition in $2-5[\mathrm{OTf}]_{2}$, whereas they do not seem to be involved in 6[OTf $]_{2}$ (Fig. S13). This is most likely related to the non-planar character of the $\pi$-core of $6[\mathrm{OTf}]_{2},{ }^{[15]}$ and is in line with the specific NICS(0) value noted for the antiaromatic P-rings (Fig. S12). More generally, the NICS(0) calculations in this series nicely follow the Clar's sextet rule for polycyclic aromatic compounds (Fig. S12).
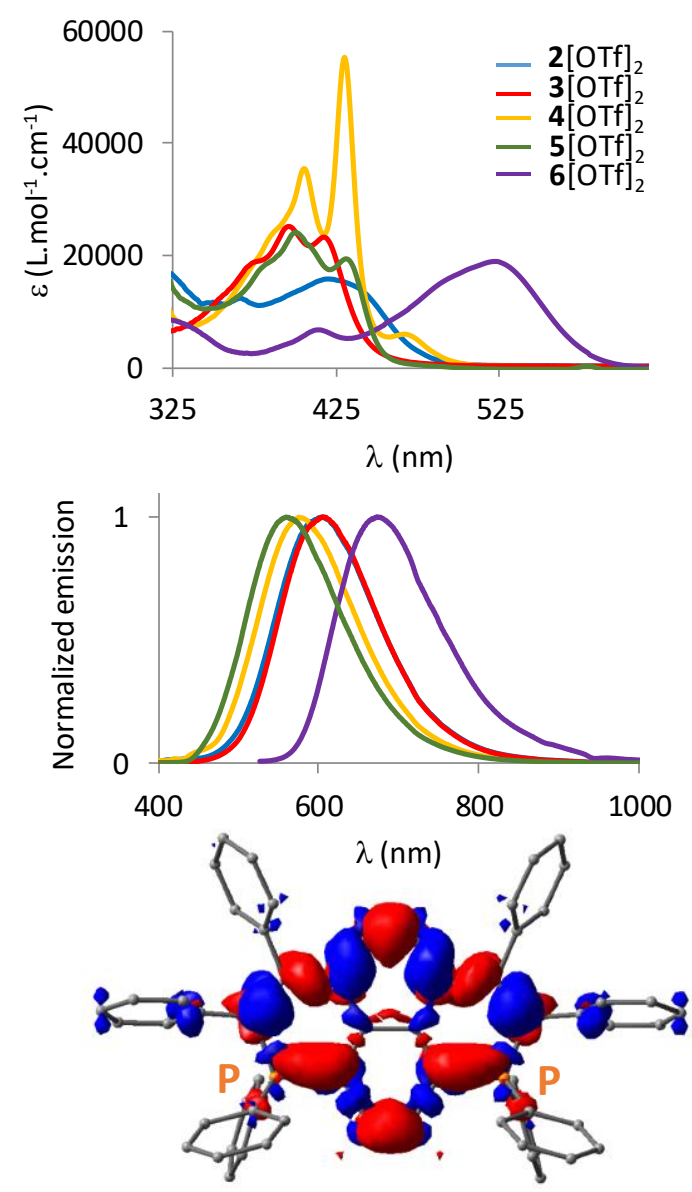

Figure 2. UV-Vis absorption (up) and normalized emission spectra (middle) of 2-6[OTf $]_{2}$ in $\mathrm{CH}_{2} \mathrm{Cl}_{2}\left(\mathrm{c}=5.10^{-6} \mathrm{M}\right)$ Electron density difference computed for $2^{2+}$ (down). The blue and red regions respectively indicate decrease and increase of density upon photon absorption (contour threshold: $810^{-4}$ ).

All derivatives display fluorescence in solution. In this case, the emission wavelengths seem more affected by the molecular structure, as a $41 \mathrm{~nm}$ shift is observed between $2[\mathrm{OTf}]_{2}$ and $5[\mathrm{OTf}]_{2}$. Again, a strong red-shift is observed for $6[\mathrm{OTf}]_{2}(\Delta \lambda(2-6)$ $=79 \mathrm{~nm}$ ). All compounds display moderate photoluminescence quantum yields PLQY $(4 \%<\phi<19 \%)$ with the lower value 
unsurprisingly obtained for the red-emitting 6[OTf $]_{2}$. Interestingly, 2-6[OTf $]_{2}$ also display fluorescence in the solid-state (Fig S2 and Table S3). It should be noted that 2-3[OTf $]_{2}$ are blue-shifted in the solid-state compared to the diluted solution while 4-6[OTf $]_{2}$ are mainly unchanged. This behaviour remains rather surprising as no clear intermolecular interactions are observed in the X-ray structures. The PLQY in solid state are higher than in solution $(11 \%<\phi<39 \%)$ with higher values for $2[\mathrm{OTf}]_{2}(39 \%)$ and $3[\mathrm{OTf}]_{2}$ (37\%). This overall increase in PLQY is attributed to the restriction of rotations of the 8 lateral phenyl rotors that are present in each molecule, and to the presence of tetrahedral $\mathrm{P}$-atom which prevent detrimental $\pi$-stacking, as confirmed by the crystallographic study. ${ }^{[16]}$

The electrochemical behavior of 2-6[OTf $]_{2}$ was investigated by cyclic voltammetry in dichloromethane solution. As anticipated by the molecular design, while no oxidation waves are observed in these conditions, all compounds display two distinctively separated reduction waves at low potential (e.g., $\operatorname{Ered}_{1}\left(2[\mathrm{OTf}]_{2}\right)=$ $-0.84 \mathrm{~V} \mathrm{vs} \mathrm{Fc}^{+} / \mathrm{Fc}$ and $\operatorname{Ered}_{2}\left(2[\mathrm{OTf}]_{2}\right)=-1.23 \mathrm{~V}$ vs $\mathrm{Fc}^{+} / \mathrm{Fc}$, Fig 3 and Table S3), suggesting the successive formation of a radical cation and a neutral specie. While the first reduction wave is fully reversible in all cases, the second reduction is quasi reversible for $2[\mathrm{OTf}]_{2}$ and $4[\mathrm{OTf}]_{2}$ (and reversible for the others). The CV traces are not altered by repeated cycles (Fig. S4). However the increase of the scan rates increases the reversibility of the waves (Fig. S5), thus highlighting the role of electron transfer rate on the stability of the reduced species. Interestingly, 2[OTf $]_{2}$ displays reduction potentials in the same range than $\mathrm{MV}[\mathrm{OTf}]_{2}$ recorded in the same conditions (Fig. S3). This clearly validates our design strategy to prepare "viologene-like" multi-stage redox systems. The effect of the $\pi$-core in the 2-6[OTf $]_{2}$ series is rather predominant with $6[\mathrm{OTf}]_{2}$ having the less cathodic potential $\left(\operatorname{Ered}_{1}\left(6[\mathrm{OTf}]_{2}\right)=-0.64 \mathrm{~V}\right.$ vs $\left.\mathrm{Fc}+/ \mathrm{Fc}\right)$ and $5[\mathrm{OTf}]_{2}$ the more cathodic $\left(\operatorname{Ered}_{1}\left(5[\mathrm{OTf}]_{2}\right)=-1.10 \mathrm{~V}\right.$ vs $\left.\mathrm{Fc}^{+} / \mathrm{Fc}\right)(\mathrm{Fig} .3)$. This trend was already observed by Würthner et al. with comparable borondoped PAHs and confirms that the highest reduction potential in these series do not correspond to the largest $\pi$-surface. ${ }^{[14]}$ However, the presence of the charged $P$ atoms renders the dicationic $\mathrm{P}$-containing $\mathrm{PAHs}$ considerably easier to reduce than their neutral B-counterparts $\left(\operatorname{Ered}_{1}\left(2[\mathrm{OTf}]_{2}\right)=-0.84 \mathrm{~V}\right.$ vs $\mathrm{Fc}^{+} / \mathrm{Fc}$ while its $\mathrm{B}$-analog displays a reduction potential Ered $_{1}=-1.46 \mathrm{~V}$ vs $\mathrm{Fc}^{+} / \mathrm{Fc}$ ). Thereby, this electrochemical study highlights the good electron accepting properties of 2-6[OTf $]_{2}$ and demonstrates that three redox states can be easily reached.

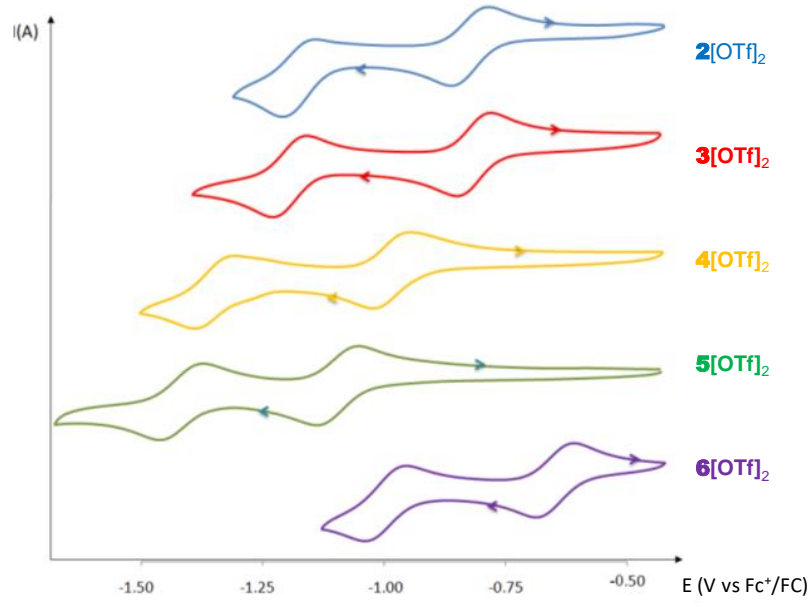

Figure 3. Cyclic voltammograms of 2-6[OTf $]_{2}\left(\mathrm{c}=5.10^{-4} \mathrm{M}\right)$ recorded in DCM $\left(\mathrm{Bu}_{4} \mathrm{NPF}_{6}(0.2 \mathrm{M}), 200 \mathrm{mVs}^{-1}\right.$, potentials vs $\left.\mathrm{Fc}^{+} / \mathrm{Fc}\right)$.

To gain more insights into the reduced forms of these derivatives, spectroelectrochemical and EPR measurements were performed on $2[\mathrm{OTf}]_{2}$, as representative compound. As illustrated Fig. 4a, each redox state of $2[\mathrm{OTf}]_{2}$ features characteristic UV-vis transitions. The absorption of the electrochemically generated radical cation $\mathbf{2}^{+}$. is characterized by the appearance of new bands in the visible and NIR regions (Fig. S7), characteristic of a highly delocalized organic radical. These new transitions subsequently disappeared upon reduction to the neutral derivative (Fig. 4a and Fig. S8). Qualitatively, the evolution of the spectra upon successive reductions is reproduced by TD-DFT (Fig S14). The absorption spectra of $\mathbf{2}^{2+}$ was further recovered upon restoring the initial potential (Fig. S9), illustrating the switching ability of this derivative. EPR was measured after chemical reduction of $\mathbf{2}^{2+}$ with $\mathrm{Zn}$ powder (Fig. 4b). This unambiguously confirmed the organic radical nature of the $2^{+}$. with $g$-factor values of 2.0032 . The hyperfine structure of the experimental EPR spectrum is well reproduced (Fig. S10) taking into account the coupling of the unpaired electron with the two equivalent phosphorus atoms and the two sets of equivalent hydrogen atoms. This demonstrates that the radical is fully delocalized on the bisphosphapyrenic scaffold. The computed spin density in the radical cation $\mathbf{2}^{+}$. (Fig. 4c) also fully supports this observation. The equalization of the $\mathrm{C}-\mathrm{C}$ bond lengths in the phosphacycle (evaluated by DFT, Table S5) are consistent with the switching between $\lambda^{4}-\mathrm{P}^{+}$cyclic phosphoniums for $2^{2+}$ and $\lambda^{5}$ phosphinine for 2. Accordingly, the aromaticity in the $P$ heterocycle increases upon reduction $\left(\operatorname{NICS}(0)\left(2^{2+}\right)=+4.6\right.$ $\operatorname{NICS}(0)(2)=-2.6$, Fig. S16). ${ }^{[17]}$ Altogether, these results illustrate that $2^{2+}$ possesses the characteristics for a new generation of electro-responsive multi-stage organic system based on organophosphorus derivatives. 

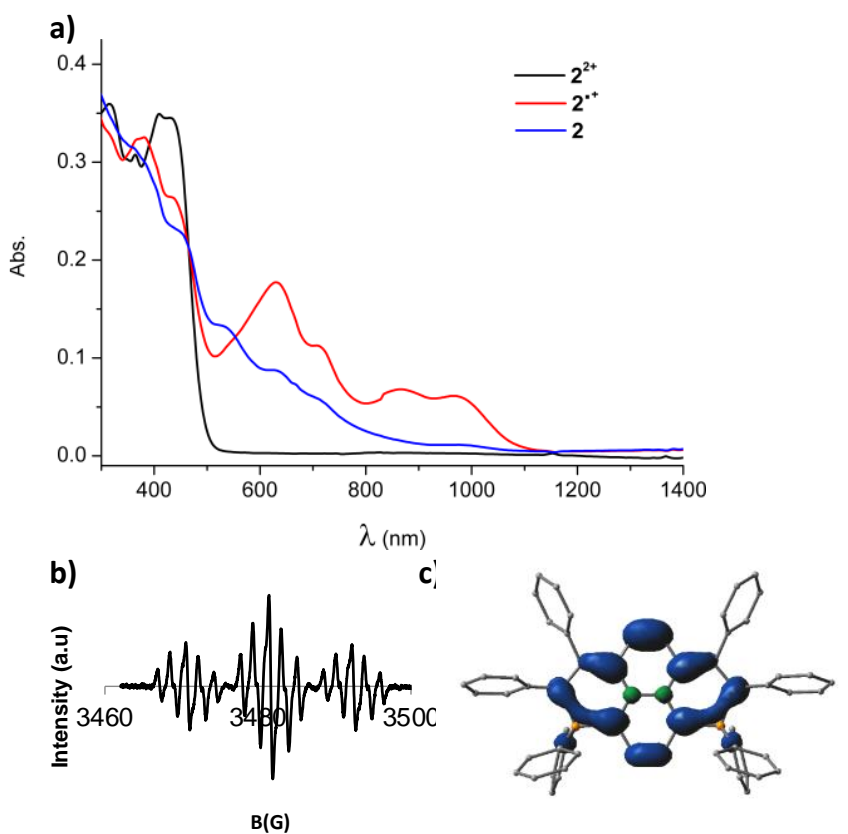

Figure 4. (a) UV/Vis/NIR absorption spectra measured during the electrochemical reduction of $2^{2+}\left(c=5.10^{-4} \mathrm{M}\right)$ in a solution of $\mathrm{Bu}_{4} \mathrm{NPF}_{6}(0.2 \mathrm{M})$ in DCM. (b) EPR spectrum of the chemically generated $2^{+.}$in DCM. (c) Spin density in $\mathbf{2}^{+\cdot}$ (contour threshold: $2.10^{-3}$ ).

In conclusion, a straightforward synthetic route to dicationic $\mathrm{P}$ containing PAH 2-6[OTf $]_{2}$ is described. The absorption and emission signatures of these compounds are changed with the $\pi$ conjugated segment, especially for the $\pi$-curved $6[\mathrm{OTf}]_{2}$. In addition, their particular structures make that 2-6[OTf $]_{2}$ possess fluorescence both in solution and in the solid-state. Furthermore, 2-6[OTf $]_{2}$ are good electron acceptors with two "viologen-like" easily accessible reduction states. $\mathbf{2}^{2+}$ was used to study the three redox states by a combined experimental (spectrolectrochemical and EPR) and theoretical study. In particular, EPR unambiguously proved the formation of the organic radical $\mathbf{2}^{+}$. This first communication highlights the great potential of these novel organophosphorus derivatives for further "viologene-like" switching applications, taking advantage of the fluorescence of 2$6[\mathrm{OTf}]_{2}$ in solution and in the solid-state. ${ }^{[18]}$ In addition, the simplicity of the synthetic approach paves the way toward the preparation of a virtually unlimited panel of redox-active $P$ derivatives based on (twisted) acenes, helicenes, nanographenes etc $^{[9,15,19]}$

\section{Acknowledgements}

This work is supported by the Ministère de la Recherche et de l'Enseignement Supérieur, the CNRS, the Région Bretagne, the French National Research Agency (ANR Heterographene ANR16-CE05-0003-01). Y. Molard and G. Taupier (Scanmat-UMS 2001) are thanked for PLQY measurements. This work used the computational resources of the CCIPL supercomputing center installed in Nantes. T. Guizouarn (ISCR) is thanked for assistance with the EPR measurements. M. Cordier and V. Dorcet (CDIFX, ISCR) are thanked for assistance with $\mathrm{X}$-ray diffraction.
[1] T. Nishinaga, (Eds), Organic Redox Systems: Synthesis, Properties, and Applications, Wiley-VCH, Weinheim, 2015.

[2] Hünig S., Berneth H. (1980) Two step reversible redox systems of the Weitz type. In: Organic Chemistry. Topics in Current Chemistry, Vol 92 Springer, Berlin, Heidelberg.

[3] K. Deuchert, S. Hünig, Angew. Chem. Int. Ed. 1978, 17, 875-886.

[4] L. Striepe, T. Baumgartner, Chem. Eur. J. 2017, 23, 16924-16940.

[5] (a) K. Madasamy, D. Velayutham, V. Suryanarayanan, M. Kathiresan, K.-C. Ho, J. Mater. Chem. C 2019, 7, 4622-4637. (b) L. Fang, M. A. Olson, D. Benítez, E. Tkatchouk, W. A. Goddard III, J. F. Stoddart, Chem. Soc. Rev. 2010, 39, 17-29.

[6] (a) T. Baumgartner, Acc. Chem. Res. 2014, 47, 1613-1622. (b) D. Joly, P. A. Bouit, M. Hissler, J. Mater. Chem. C 2016, 4, 3686-3698. (c) A. I. Arkhypchuk, E. Mijangos, R. Lomoth, S. Ott, Chem. Eur. J. 2014, 20 , 16083-16087. (d) J. C.-H. Chan, W. H. Lam, H.-L. Wong, W.-T. Wong, V. W.-W. Yam, Angew. Chem. Int. Ed. 2013, 52, 11504-11508. (e) M. Stolar, J. Borau-Garcia, M. Toonen, T. Baumgartner, J. Am. Chem. Soc. 2015, 137, 3366-3371. (f) T. Baumgartner, R. Réau, Chem. Rev. 2006, 106, 4681-4727.

[7] Various derivatives fulfil the structural requirements of Scheme 1, but do not show chemical/redox stability allowing the multi-stage redox processing even if radical cations could be isolated: (a) F. Murakami, $\mathrm{S}$ Sasaki, M. Yoshifuji, Angew. Chem. Int. Ed. 2002, 41, 2574-2576. (b) F. Biaso, T. Cantat, N. Mézailles, L. Ricard, P. Le Floch, M. Geoffroy, Angew. Chem. Int. Ed. 2006, 45, 7036-7039. (c) P. Federmann, H. K. Wagner, P. W. Antoni, J.-M. Mörsdorf, J. L. Pérez Lustres, H. Wadepohl, M. Motzkus, J. Ballmann, Org. Lett. 2019, 21, 2033-2038. (d) P Hindenberg, F. Rominger, C. Romero-Nieto, Chem. Eur. J. 2019, 25, 13146-13151

[8] The compound 7[OTf $]_{2}$ described by Anslyn et al. (S. Nieto, P. Metola, V. M. Lynch, E. V. Anslyn, Organometallics 2008, 27, 3608-3610) was recently studied for its redox properties but only the first reduction was described $(\mathrm{H}$. Cheng, X. Wang, L. Chang, Y. Chen, L. Chu, Z. Zuo Science Bulletin 2019, 64, 1896-1901. The second reduction is here described in Fig. S6

[9] M. Stępień, E. Gońka, M. Żyła, N. Sprutta, Chem. Rev. 2016, 117, 34793716 .

[10] $\sigma$ represents the coordination number and $\lambda$ the valence of the $P$-atom, see : Multiple Bonds and Low Coordination in Phosphorus Chemistry; M. Regitz, O. Scherer (Eds.) Thieme: Stuttgart, 1990.

[11] Q. Ge, J. Zong, B. Li, B. Wang, Org. Lett. 2017, 19, 6670-6673.

[12] (a) A. Belyaev, Y.-T. Chen, S.-H. Su, Y.-J. Tseng, A. J. Karttunen, S. P. Tunik, P.-T. Chou, I. O. Koshevoy, Chem. Commun. 2017, 53, 1095410957. (b) A. Belyaev, Y.-T. Chen, Z.-Y. Liu, P. Hindenberg, C.-H. Wu, P.-T. Chou, C. Romero-Nieto, I. O. Koshevoy, Chem. Eur. J. 2019, 25, 6332-6341. (c) L. Qiu, W. Hu, D. Wu, Z. Duan, F. Mathey, Org. Lett. 2018, 20, 7821-7824.

[13] M. A. Majewski, M. Stępień, Angew. Chem. Int. Ed. 2019, 58, 86-116.

[14] This trend was already observed by Würthner et al. in their study of comparable boron-doped PAHs: J. M. Farrell, C. Mützel, D. Bialas, M. Rudolf, K. Menekse, A.-M. Krause, M. Stolte, F. Würthner, J. Am. Chem. Soc. 2019, 141, 9096-9104.

[15] A. Bedi, O. Gidron, Acc. Chem. Res. 2019, 52, 2482-2490.

[16] Y. Hong, J. W. Y. Lam, B. Z. Tang, Chem. Soc. Rev. 2011, 40, 53615388.

[17] (a) N. Hashimoto, R. Umano, Y. Ochi, K. Shimahara, J. Nakamura, S Mori, H. Ohta, Y. Watanabe, M. Hayashi, J. Am. Chem. Soc. 2018, 140, 2046-2049. (b) Z. Benkö, L. Nyulaszi, Top. Heterocycl. Chem. 2009, 19, 27-83.

[18] Viologen derivatives are only luminescent under specific conditions: $M$ Freitag, L. Gundlach, P. Piotrowiak, E. Galoppini, J. Am. Chem. Soc. 2012, 134, 3358-3366

[19] (a) K. Dhbaibi, L. Favereau, J. Crassous, Chem. Rev. 2019, 119, 88468953. (b) T. Y. Gopalakrishna, W. Zeng, X. Lu, J. Wu, Chem. Commun. 2018, 54, 2186-2199. (c) P.-A. Bouit, A. Escande, R. Szücs, D. Szieberth, C. Lescop, L. Nyulászi, M. Hissler, R. Réau, J. Am. Chem. Soc. 2012, $134,6524-6527$ 
